\title{
Determination of a High Potential Barrier Hindering Internal Rotation from the Ground State Spectrum
}

\section{The Methylbarrier of Ethylchloride}

\author{
W. Stahl and H. Dreizler \\ Abteilung Chemische Physik im Institut für Physikalische Chemie der Universität Kiel \\ M. Hayashi \\ Hiroshima University, Department of Chemistry, Faculty of Science, Higashisenda-machi, \\ Hiroshima-City-Japan
}

Z. Naturforsch. 38a, 1010-1014 (1983); received May 25, 1983

\begin{abstract}
We present an analysis of the rotational spectrum of ethylchloride $-{ }^{35} \mathrm{Cl}$ in the ground state. The ${ }^{35} \mathrm{Cl}$-hfs analysis was extended and the barrier to internal rotation determined from narrow splittings of high $J$-transitions.
\end{abstract}

\section{Introduction}

With the higher resolution of microwave Fourier transform (MWFT) spectroscopy it is possible to resolve fine structures, which could not be measured before [1-3].

We noticed that the barrier to internal rotation $V_{3}$ of ethylchloride, $\mathrm{CH}_{3} \mathrm{CH}_{2} \mathrm{Cl}$, was determined [4, 5] from two splittings in the first torsional excited state. As there is in some molecules an interaction of the internal rotation and other vibrations [6] we thought it interesting to determine the barrier from ground state measurements.

\section{Experimental}

The sample was purchased from FLUKA with a $98 \%$ purity. The spectra were recorded by a conventional Starkspectrometer $[7,8]$ in the region of $8-40 \mathrm{GHz}$ with temperatures from -30 to $-45^{\circ} \mathrm{C}$ and pressures between 4 and $15 \mathrm{mT}$ Torr and for high resolution with a MWFT-Spectrometer from 8 to $18 \mathrm{GHz}$ [1] with temperatures from -60 to $-75^{\circ} \mathrm{C}$ and pressures between 0.3 and $1.5 \mathrm{mTorr}$. The measuring errors are $50 \mathrm{kHz}$ and $10 \mathrm{kHz}$ respectively.

\section{Measurements and Analysis}

As the barrier $V_{3}$ is rather high, splittings could only be observed for high $J$ lines within the range of our MWFT spectrometer. For correct assignment a

Reprint requests to Prof. Dr. H. Dreizler, Institut für Physikalische Chemie der Christian-Albrechts-Universität Kiel, Olshausenstr. 40, D-2300 Kiel. centrifugal distortion and extended chlorine hfs analysis was necessary.

In Table 1 we give the lines measured by us and by other authors [5]. They are marked by *. With FT we indicate MWFT measurements. In Fig. 1 we give a multiplet pattern.

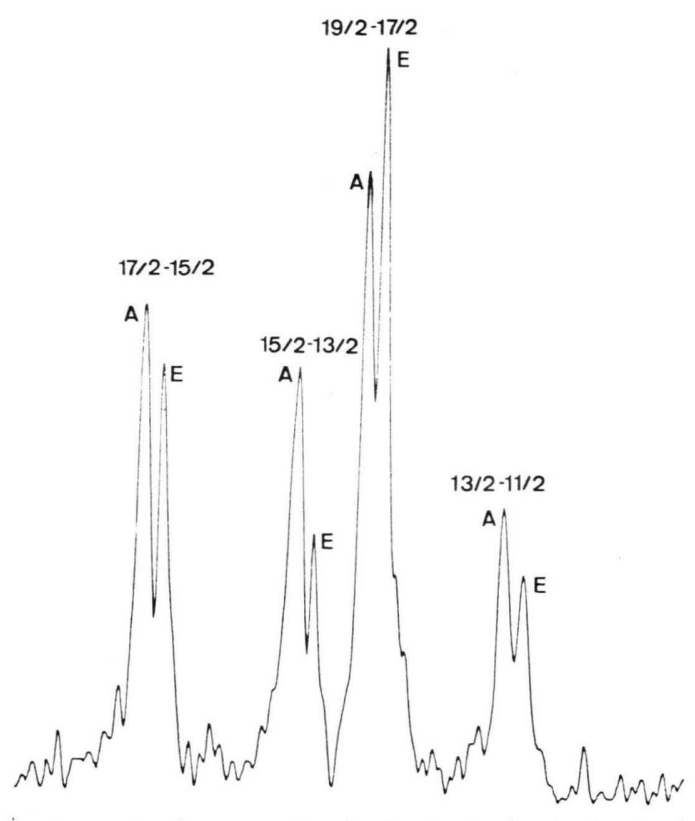

Fig. 1. A section of $1.5 \mathrm{MHz}$ of the rotational spectrum of $\mathrm{CH}_{3} \mathrm{CH}_{2}{ }^{35} \mathrm{Cl}$ showing the $8_{17}-72$ line with ${ }^{35} \mathrm{Cl}-\mathrm{hfs}$ and internal rotation (A, E-species) splitting. Pressure $0.4 \mathrm{mT}$, temperature $-70^{\circ} \mathrm{C}$. Transient emission signal was recorded with a sample interval of $50 \mathrm{~ns}$ and 1024 data points. Measuring time $3 \mathrm{~min} 256000$ averaging cycles. Prior to Fourier transformation the time domain signal was extended to 4096 points by zeros. Spectral point distance $5 \mathrm{kHz}$.

0340-4811/83/0900-1010\$01.3 0/0. - Please order a reprint rather than making your own copy.

Dieses Werk wurde im Jahr 2013 vom Verlag Zeitschrift für Naturforschung in Zusammenarbeit mit der Max-Planck-Gesellschaft zur Förderung der Wissenschaften e.V. digitalisiert und unter folgender Lizenz veröffentlicht: Creative Commons Namensnennung-Keine Bearbeitung 3.0 Deutschland Lizenz.

Zum 01.01.2015 ist eine Anpassung der Lizenzbedingungen (Entfall de Creative Commons Lizenzbedingung „Keine Bearbeitung“) beabsichtigt um eine Nachnutzung auch im Rahmen zukünftiger wissenschaftlicher Nutzungsformen zu ermöglichen.
This work has been digitalized and published in 2013 by Verlag Zeitschrift für Naturforschung in cooperation with the Max Planck Society for the Advancement of Science under a Creative Commons Attribution-NoDerivs 3.0 Germany License. 
Table 1. Measured lines $\left(v_{\exp }\right)$ of ethylchloride $-{ }^{35} \mathrm{Cl}[\mathrm{MHz}] . v_{\text {ave }}$ : averaged by hfs-shifts, $\Delta v_{\text {exp }}$ : experimental hfs-shifts, $\Delta v_{\text {calc }}$ : calculated hfs-shifts, FT: measured by MWFT-spectroscopy, ${ }^{*}$ : measured by other authors [5].

\begin{tabular}{|c|c|c|c|c|c|c|c|c|c|c|c|c|c|c|c|c|c|c|c|c|c|c|c|}
\hline$J$ & $K-k$ & $\alpha+$ & $\mathrm{J}$ & $k-P$ & & $2 F$ & $2 F^{\prime}$ & $\nu \exp$ & Lave & $\Delta \nu \exp$ & $\Delta \nu$ calc & J & k- & $k+$ & $\mathrm{J}^{\prime}$ & $k-’ k$ & $k+1$ & $2 F$ & $2 F^{\prime}$ & $\nu \exp$ & Lave & $\Delta \nu \exp$ & $\Delta \nu c a l c$ \\
\hline 1 & 0 & 1 & 0 & 0 & 0 & $\begin{array}{l}3 \\
5 \\
1 \\
1\end{array}$ & $\begin{array}{l}3 \\
3 \\
3\end{array}$ & $\begin{array}{l}10446.081 \\
10458.478 \\
10468.191\end{array}$ & 10455.943 & $\begin{array}{r}-9.862 \\
2.535 \\
12.248\end{array}$ & $\begin{array}{r}-9.853 \\
2.463 \\
12.317\end{array}$ & 9 & 1 & 8 & 8 & 2 & 7 & $\begin{array}{l}15 \\
21 \\
17 \\
19\end{array}$ & $\begin{array}{l}13 \\
19 \\
15 \\
17\end{array}$ & $\begin{array}{l}27279.737 \\
27280.091 \\
27280.675 \\
27281.014\end{array}$ & 27280.409 & $\begin{array}{r}-0.672 \\
-0.318 \\
0.266 \\
0.605\end{array}$ & $\begin{array}{r}-0.675 \\
-0.324 \\
0.266 \\
0.615\end{array}$ \\
\hline \multirow[t]{3}{*}{${ }^{2}$} & 0 & 2 & 1 & 0 & 1 & $\begin{array}{l}3 \\
5 \\
5\end{array}$ & $\begin{array}{l}1 \\
5 \\
3\end{array}$ & $\begin{array}{l}20891.45 \\
20892.41\end{array}$ & 20903.780 & $\begin{array}{l}-12.330 \\
-11.370\end{array}$ & $\begin{array}{l}-12.317 \\
-11.300\end{array}$ & 8 & 3 & 6 & 9 & 2 & 7 & 13 & 15) & 34434.938 & 34435.945 & -1.007 & -1.066 \\
\hline & & & & & & 7 & 5 & 20904.87 & & 1.090 & 1.044 & & & & & & & $\begin{array}{l}19 \\
15\end{array}$ & $\begin{array}{l}21) \\
17)\end{array}$ & 4604.000 & & & \\
\hline & & & & & & 3 & 3 & 20913.69 & & 9.910 & 9.853 & & & & & & & 17 & $\left.{ }_{19}\right\}$ & 34436.947 & & 1.002 & 1.062 \\
\hline \multirow[t]{4}{*}{3} & 0 & 3 & 2 & 0 & 2 & $\begin{array}{l}7 \\
5\end{array}$ & $\begin{array}{l}7 \\
3\end{array}$ & 31323.373 & 31335.232 & -11.859 & -11.836 & 10 & 3 & 8 & 11 & 2 & 9 & 23 & $25)$ & $\int 11372.841$ & 11373.241 & & \\
\hline & & & & & & $\begin{array}{l}3 \\
7\end{array}$ & $\left.\begin{array}{l}1 \\
5\end{array}\right\}$ & 31332.765 & & -2.467 & -2.450 & FT & & & & & & 17 & $\left.{ }_{19}\right\}$ & $\left\{\begin{array}{l}11372.898 \\
11372.923\end{array}\right.$ & & -0.354 & -0.356 \\
\hline & & & & & & 9 & $\left.{ }_{7}^{3}\right\}$ & 31335.786 & & 0.554 & 0.576 & & & & & & & $\begin{array}{l}21 \\
19\end{array}$ & $\left.\begin{array}{l}23 \\
21\end{array}\right\}$ & $\left\{\begin{array}{l}11373.571 \\
11373.629 \\
11373.651\end{array}\right.$ & & 0.376 & 0.369 \\
\hline & & & & & & $\frac{5}{3}$ & $\frac{5}{3}$ & $\begin{array}{l}31341.571 \\
31345.261\end{array}$ & & $\begin{array}{r}6.339 \\
10.029\end{array}$ & $\begin{array}{l}6.346 \\
9.962\end{array}$ & 15 & 2 & 13 & 14 & 31 & 12 & 27 & $25\}$ & 38559.908 & 38560.303 & -0.395 & -0.405 \\
\hline \multirow{3}{*}{$*^{2}$} & 1 & 1 & 1 & 1 & 0 & 5 & 3 & 21433.79 & 21443.375 & -9.585 & -9.562 & & & & & & & $\begin{array}{l}33 \\
29\end{array}$ & $\begin{array}{l}315 \\
27)\end{array}$ & 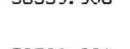 & & -0.070 & -0.405 \\
\hline & & & & & & $\begin{array}{l}3 \\
5 \\
7 \\
3\end{array}$ & $\begin{array}{l}3 \\
5 \\
5 \\
1\end{array}$ & $\begin{array}{l}21436.27 \\
21442.73 \\
21446.21 \\
21452.27\end{array}$ & & $\begin{array}{r}-7.105 \\
-0.645 \\
2.835 \\
8.895\end{array}$ & $\begin{array}{r}-7.135 \\
-0.644 \\
2.755 \\
8.918\end{array}$ & & & & & & & 31 & $\left.{ }_{29}\right\}$ & 38560.684 & & 0.381 & 0.391 \\
\hline & & & & & & 1 & 1 & 21455.62 & & 12.245 & $\begin{array}{r}8.918 \\
12.317\end{array}$ & 23 & 6 & 18 & 24 & 51 & 19 & 43 & 45) & 34152.275 & 34152.633 & -0.358 & -0.367 \\
\hline \multirow[t]{4}{*}{3} & 1 & 2 & 2 & 1 & 1 & $\begin{array}{l}7 \\
7\end{array}$ & $\begin{array}{l}7 \\
5\end{array}$ & $\begin{array}{l}32154.405 \\
32157.814\end{array}$ & 32159.721 & $\begin{array}{l}-5.316 \\
-1.907\end{array}$ & $\begin{array}{l}-5.301 \\
-1.902\end{array}$ & & & & & & & $\begin{array}{l}49 \\
45\end{array}$ & $\begin{array}{l}51\} \\
47\}\end{array}$ & & & & \\
\hline & & & & & & $\begin{array}{l}5 \\
9\end{array}$ & $\begin{array}{l}3 \\
7\end{array}$ & 32158.431 & & -1.290 & -1.299 & & & & & & & 47 & 49 & 34152.992 & & 0.359 & 0.367 \\
\hline & & & & & & 5 & 5 & 32160.906 & & 1.185 & 1. 161 & $\begin{array}{l}25 \\
\text { FT }\end{array}$ & 6 & 20 & 26 & 52 & 21 & 47 & 49) & $\int 12512.736$ & 12513.054 & -0.285 & -0.283 \\
\hline & & & & & & $\begin{array}{l}3 \\
3\end{array}$ & $\frac{1}{3}$ & $\begin{array}{l}32161.484 \\
32164.935\end{array}$ & & $\begin{array}{l}1.763 \\
5.214\end{array}$ & $\begin{array}{l}1.797 \\
5.196\end{array}$ & & & & & & & $\begin{array}{l}53 \\
49\end{array}$ & $\begin{array}{l}55\} \\
51\}\end{array}$ & 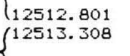 & & - & 0.200 \\
\hline \multirow[t]{3}{*}{${ }^{2}$} & 1 & 2 & 1 & 1 & 1 & $\begin{array}{l}5 \\
5 \\
7\end{array}$ & $\begin{array}{l}3 \\
5 \\
7\end{array}$ & $\begin{array}{l}20371.50 \\
20374.88\end{array}$ & 20380.574 & $\begin{array}{l}-9.074 \\
-5.694\end{array}$ & $\begin{array}{l}-9.089 \\
-5.690\end{array}$ & & & & & & & 51 & 53 & 12513.373 & & 0.287 & 0.284 \\
\hline & & & & & & $\begin{array}{l}3 \\
7\end{array}$ & $\begin{array}{l}3 \\
5\end{array}$ & $\begin{array}{l}20377.89 \\
20383.79\end{array}$ & & $\begin{array}{r}2.684 \\
3.216\end{array}$ & $\begin{array}{r}2.719 \\
3.228\end{array}$ & $\begin{array}{l}28 \\
\mathrm{FT}\end{array}$ & 5 & 23 & 27 & 62 & 22 & 57 & $55)$ & $\int 9350.536$ & 9350.784 & -0.216 & -0.212 \\
\hline & & & & & & 1 & 1 & 20392.86 & & 12.286 & 12.317 & & & & & & & $\begin{array}{l}55 \\
59\end{array}$ & $\begin{array}{l}53 \int \\
57)\end{array}$ & $\begin{array}{l}\text { (9350. } 600 \\
9350.967\end{array}$ & & -0.210 & -0.212 \\
\hline \multirow[t]{4}{*}{3} & 1 & 3 & 2 & 1 & 2 & $\begin{array}{l}7 \\
5 \\
7\end{array}$ & $\begin{array}{l}7 \\
3 \\
5\end{array}$ & $\begin{array}{l}30555.108 \\
30563.261 \\
30564.018\end{array}$ & 30565.658 & $\begin{array}{l}-10.550 \\
-2.397 \\
-1.640\end{array}$ & $\begin{array}{l}-10.557 \\
-2.403 \\
-1.639\end{array}$ & & & & & & & 53 & $\left.{ }_{51}^{37}\right\}$ & $\left\{\begin{array}{l}9350.961 \\
9351.031\end{array}\right.$ & & 0.215 & 0.211 \\
\hline & & & & & & 3 & 1 & 30566.361 & & 0.703 & 0.693 & 30 & 5 & 25 & 29 & 62 & 24 & 59 & 57) & & 31507.863 & & \\
\hline & & & & & & $\begin{array}{l}9 \\
5 \\
7\end{array}$ & $\begin{array}{l}7 \\
5 \\
7\end{array}$ & $\begin{array}{l}30567.115 \\
30569.604\end{array}$ & & $\begin{array}{l}1.457 \\
3.946\end{array}$ & $\begin{array}{l}1.457 \\
3.967\end{array}$ & & & & & & & 61 & $\left.{ }_{59}\right\}$ & 31507.741 & & -0.122 & -0.147 \\
\hline & & & & & & 3 & 3 & 30575.268 & & 9.610 & 9.611 & & & & & & & 57 & $55\}$ & & & & \\
\hline \multirow[t]{2}{*}{1} & 1 & 1 & 0 & 0 & 0 & $\frac{1}{5}$ & $\begin{array}{l}3 \\
3 \\
7\end{array}$ & $\begin{array}{l}36296.101 \\
36298.827\end{array}$ & 36299.500 & $\begin{array}{l}-3.399 \\
-0.673\end{array}$ & $\begin{array}{l}-3.399 \\
-0.680\end{array}$ & & & & & & & 63 & $61\}$ & 31507.984 & & 0.121 & 0.147 \\
\hline & & & & & & 3 & 3 & 36302.213 & & 2.713 & 2.719 & 33 & 8 & 26 & 34 & 72 & 27 & 63 & $\left.{ }^{65}\right\}$ & 33243.974 & 33244.404 & -0.430 & -0.244 \\
\hline \multirow[t]{4}{*}{$\begin{array}{r}4 \\
F T\end{array}$} & 0 & 4 & 3 & 1 & 3 & 7 & 5 & $\begin{array}{r}17190.190 \\
17190.754\end{array}$ & 17191.388 & -1.198 & -1.186 & & & & & & & $\begin{array}{l}69 \\
65\end{array}$ & $\begin{array}{l}713 \\
677\end{array}$ & 300240.374 & & -0.430 & -0.244 \\
\hline & & & & & & 5 & 3 & 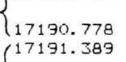 & & -0.622 & -0.640 & & & & & & & 67 & $\left.{ }_{69}\right\}$ & 33244.834 & & 0.430 & 0.244 \\
\hline & & & & & & 9 & 7 & $\left\{\begin{array}{l}17191.408 \\
10\end{array}\right.$ & & 0.011 & 0.016 & $\begin{array}{l}35 \\
\mathrm{FT}\end{array}$ & 8 & 28 & 36 & 72 & 29 & & & $\begin{array}{l}11704.656 \\
11704.689\end{array}$ & 11704.880 & & \\
\hline & & & & & & 11 & 9 & 17191.950 & & 0.562 & 0.563 & & & & & & & & & $\begin{array}{l}11705.073 \\
11705.100\end{array}$ & & & \\
\hline \multirow[t]{3}{*}{5} & 0 & 5 & 4 & 1 & 4 & $\begin{array}{l}7 \\
9\end{array}$ & $\begin{array}{l}5 \\
7\end{array}$ & $\begin{array}{l}28562.449 \\
28563.014\end{array}$ & 28563.450 & $\begin{array}{l}-1.001 \\
-0.436\end{array}$ & $\begin{array}{l}-1.016 \\
-0.440\end{array}$ & 40 & 7 & 33 & 39 & 83 & 32 & 79 & 77) & & 31753.143 & & \\
\hline & & & & & & $\begin{array}{l}13 \\
11\end{array}$ & $\begin{array}{r}11 \\
9\end{array}$ & $\begin{array}{l}28563.498 \\
28564.073\end{array}$ & & $\begin{array}{l}0.048 \\
0.623\end{array}$ & $\begin{array}{l}0.057 \\
0.633\end{array}$ & & & & & & & 81 & $\left.{ }_{79}\right\}$ & 31753.021 & & -0.122 & \\
\hline & 2 & 2 & 4 & 1 & 3 & 3 & 5 & 33860.833 & 33866.391 & -5.558 & - 5.538 & & & & & & & 77 & $75\}$ & 31753,264 & & 0,121 & \\
\hline \multirow{2}{*}{3} & & & & & & $\begin{array}{l}7 \\
5\end{array}$ & $\begin{array}{r}11 \\
7\end{array}$ & $\begin{array}{l}33863.560 \\
33868.626\end{array}$ & & $\begin{array}{r}-2.831 \\
2.235\end{array}$ & $\begin{array}{r}-2.819 \\
2.215\end{array}$ & & & & & & & 83 & $\left.{ }_{81}\right\}$ & 31753.264 & & 0.121 & \\
\hline & & & & & & 7 & 9 & 33871.337 & & 4.948 & 4.934 & 13 & 4 & 10 & 14 & 3 & 11 & 23 & $\left.{ }^{25}\right\}$ & 34764.025 & 34764.690 & -0.665 & -0.666 \\
\hline \multirow[t]{5}{*}{5} & 2 & 4 & 6 & 1 & 5 & 13 & $15\}$ & 10113848 & 10114.508 & $660<-2>0$ & 657 & & & & & & & 29 & $\begin{array}{l}31 \\
27)\end{array}$ & & & & \\
\hline & & & & & & $?$ & ${ }_{9}^{9}$ & 10113.848 & & 660 & & & & & & & & 25 & $27\}$ & 34765.355 & & 0.665 & 0.666 \\
\hline & & & & & & $\begin{array}{r}11 \\
9\end{array}$ & $\left.\begin{array}{l}13 \\
11\end{array}\right\}$ & 10115.229 & & 0.721 & 0.718 & $\begin{array}{l}15 \\
\mathrm{FT}\end{array}$ & 4 & 12 & 16 & 3 & 13 & $\begin{array}{l}27 \\
23\end{array}$ & $\begin{array}{l}29) \\
21)\end{array}$ & $(12589.241$ & 12589.660 & -0.382 & -0.380 \\
\hline & & & & & & & & & & & & & & & & & & $\begin{array}{l}29 \\
25\end{array}$ & $\begin{array}{l}\text { 27) } \\
23)\end{array}$ & $\begin{array}{l}12589.314 \\
12590.006\end{array}$ & & & \\
\hline & & & & & & & & & & & & & & & & & & 27 & $\left.{ }_{25}\right\}$ & 12590.082 & & 0.384 & 0.383 \\
\hline \multirow[t]{5}{*}{$\begin{array}{r}8 \\
F T\end{array}$} & 1 & 7 & 7 & 2 & 6 & 13 & 11 & $\left\{\begin{array}{l}14591.258 \\
\end{array}\right.$ & 14591.702 & -0.420 & -0.444 & 18 & 3 & 15 & 17 & 4 & 14 & 35 & $33\}$ & 10328.093 & 10328.206 & -0.113 & -0.151 \\
\hline & & & & & & & & $\begin{array}{l}14591.306 \\
\int_{14591.578}\end{array}$ & & & & & & & & & & $\begin{array}{l}37 \\
33\end{array}$ & $\begin{array}{l}35 \\
31\end{array}$ & 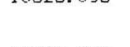 & & 1.113 & \\
\hline & & & & & & 19 & 17 & 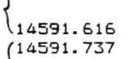 & & -0.105 & -0.116 & & & & & & & 39 & $\left.\begin{array}{l}31 \\
37\end{array}\right\}$ & 10328.315 & & 0.109 & 0.147 \\
\hline & & & & & & 15 & 13 & $\left\{\begin{array}{l}14591.771\end{array}\right.$ & & -0.052 & 0.046 & 20 & 3 & 17 & 19 & 41 & 16 & $\begin{array}{l}43 \\
41\end{array}$ & $\begin{array}{l}41 \\
39\end{array}$ & & 34262.347 & & \\
\hline & & & & & & 17 & 15 & $\left\{\begin{array}{l}14592.078 \\
14592.119\end{array}\right.$ & & 0.397 & 0.374 & & & & & & & $\begin{array}{l}39 \\
37\end{array}$ & $\left.\begin{array}{l}37 \\
35\end{array}\right\}$ & 34262.947 & & 0.000 & 0.000 \\
\hline
\end{tabular}


Table 1 (continued).

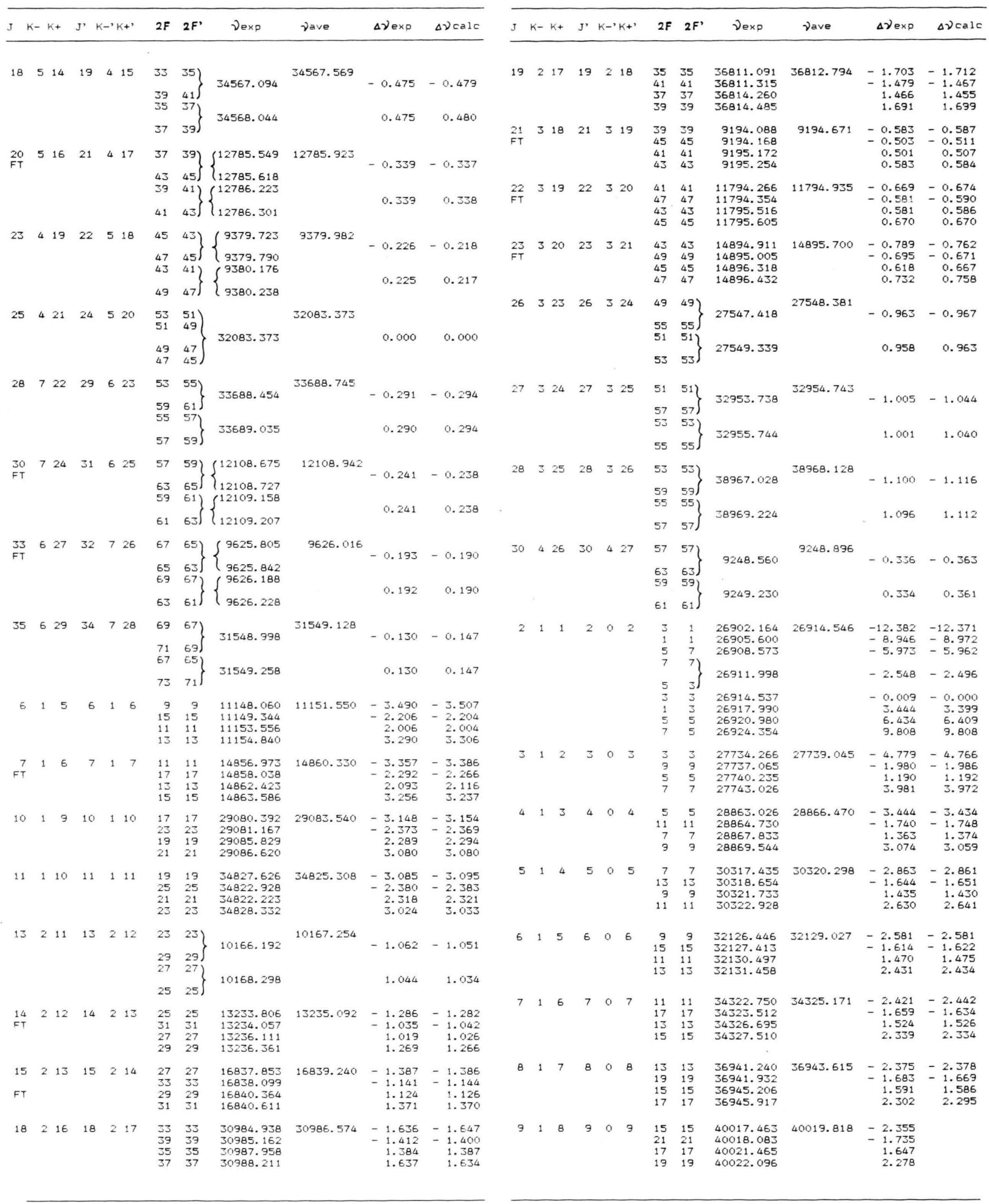


Table 2. Rotational [MHz] and centrifugal distortion $[\mathrm{kHz}]$ constants according to Watson's S-reduction. $|(a, b)|$ highest correlation, $\sigma$ : standard deviation of the fit $[\mathrm{kHz}]$.

\begin{tabular}{lclc}
\hline$A$ & $31337.406(34)$ & $\mathfrak{U}$ & $31337.413(34)$ \\
$B$ & $5493.699(6)$ & $\mathfrak{B}$ & $5493.682(6)$ \\
$C$ & $4962.276(6)$ & $\mathfrak{C}$ & $4962.261(6)$ \\
$D_{J}$ & $3.40(5)$ & $\tau_{a a a a}$ & $-999(4)$ \\
$D_{J K}$ & $-22.3(8)$ & $\tau_{b b b b}$ & $18.1(2)$ \\
$D_{K}$ & $268(2)$ & $\tau_{c c c c}$ & $-9.4(2)$ \\
$d_{1}$ & $-0.540(2)$ & $\tau_{1}$ & $49(4)$ \\
$d_{2}$ & $-0.018(1)$ & $\tau_{2}$ & $-87(23)$ \\
$\sigma$ & 142 & & \\
$\left|\left(D_{J}, D_{J K}\right)\right|$ & 0.999 & & \\
\hline
\end{tabular}

The centrifugal distortion and hfs analysis were made independently. For the centrifugal distortion analysis unsplit lines $v_{\text {ave }}$ corrected for hfs were used. For the hfs analysis it was assumed that the components of one transition are equally shifted by centrifugal distortion. In Table 2 we give the rotational and centrifugal distortion constants according to the fourth order Hamiltonian of Watson's S-reduction [9]. The standard deviation of the fit is $142 \mathrm{kHz}$, the highest correlation is $\mid\left(D_{J}, D_{J K}\right)=0.999$.

Table 3. Chlorine hfs coupling constants [MHz] of $\mathrm{CH}_{3} \mathrm{CH}_{2}{ }^{35} \mathrm{Cl}$ calculated with different selections of lines (column $1 J$ to 23 and column $2 J$ to 3 ) and first order approximation. Column 3 gives values from lines up to $J=3$ calculated with diagonalisation [10]. Standard errors are given. $\left(\chi^{+}, \chi^{-}\right) \mid$correlation coefficient. Column 4 is calculated from [5].

\begin{tabular}{lrrrr}
\hline$\chi^{+}$ & $49.267(52)$ & $49.21(12)$ & $49.241(47)$ & $49.20(10)$ \\
$\chi^{-}$ & $-22.078(30)$ & $-22.09(18)$ & $-22.100(72)$ & $-22.04(21)$ \\
$\left(\chi^{+}, \chi^{-}\right) \mid$ & 0.014 & 0.203 & 0.375 & - \\
$\chi_{a a}$ & $-49.267(52)$ & $-49.21(12)$ & $-49.241(47)$ & $-49.20(10)$ \\
$\chi_{b b}$ & $13.595(41)$ & $13.56(15)$ & $13.570(60)$ & $13.58(16)$ \\
$\chi_{c c}$ & $35.673(41)$ & $35.65(15)$ & $35.670(60)$ & $35.62(16)$ \\
\hline
\end{tabular}

Table 4. Internal Rotation splittings $[\mathrm{kHz}]$ of $\mathrm{CH}_{3} \mathrm{CH}_{2}{ }^{35} \mathrm{Cl}$ averaged over hfs components.

\begin{tabular}{rrrrrrll}
\hline$J$ & $K^{-}$ & $K^{+}$ & $J^{\prime}$ & $K^{-\prime}$ & $K^{+\prime}$ & $\Delta v_{\text {obs }}$ & $\Delta v_{\text {calc }}$ \\
\hline 8 & 1 & 7 & 7 & 2 & 6 & 40 & 36.3 \\
10 & 3 & 8 & 11 & 2 & 9 & 69 & 63.7 \\
15 & 4 & 12 & 16 & 3 & 13 & 75 & 76.4 \\
18 & 3 & 15 & 17 & 4 & 14 & 68 & 69.8 \\
20 & 5 & 16 & 21 & 4 & 17 & 74 & 76.4 \\
23 & 4 & 19 & 22 & 5 & 18 & 67 & 69.2 \\
25 & 6 & 20 & 26 & 5 & 21 & 65 & 65.7 \\
28 & 5 & 23 & 27 & 6 & 22 & 64 & 57.9 \\
30 & 7 & 24 & 31 & 6 & 25 & 51 & 47.9 \\
33 & 6 & 27 & 32 & 7 & 26 & 38 & 40.0 \\
35 & 8 & 28 & 36 & 7 & 29 & 30 & 28.0 \\
\hline
\end{tabular}

We also tried sixth order centrifugal distortion analysis, which improves the fit. But the constants are poorly determined, and many correlation coefficients are high.

The hfs analysis was based on lines with $J$ up to 23. We omitted lines split by internal rotation. In Table 3 we give the quadrupole coupling constants $\chi_{g g}, g=a, b, c$, calculated with first order theory. A mean shift of $3.14 \mathrm{MHz}$ was fitted with a standard deviation of $0.027 \mathrm{MHz}$. To check the influence of centrifugal distortion we give also the $\chi_{g g}$ determined from lines with $J$ up to 3 . The approximation

Table 5. Internal rotation parameters of $\mathrm{CH}_{3} \mathrm{CH}_{2}{ }^{35} \mathrm{Cl}$. Standard errors in brackets. Assumptions in square brackets.

\begin{tabular}{lcccc}
\hline$w_{1}(s)$ & $-6.05(11) \cdot 10^{-7}$ & $-5.88(14) \cdot 10^{-7}$ & $-5.87(15) \cdot 10^{-7}$ & - \\
$\lambda_{a}$ & {$[0.7317]$} & {$[0.7317]$} & {$[0.7317]$} & {$[0.7317]$} \\
$\lambda_{b}$ & {$[0.6816]$} & {$[0.6816]$} & {$[0.6816]$} & {$[0.6816]$} \\
$I_{\alpha}\left[\mathrm{amu} \AA^{2}\right]$ & $3.246(15)$ & {$[3.1613]$} & {$[3.155]$} & {$[3.1613]$} \\
$\left|\left(w_{1}(s), I_{\alpha}\right)\right|$ & 0.383 & - & - & - \\
$\overline{\Delta v_{\exp }[\mathrm{kHz}]}$ & 58.3 & 58.3 & 58.3 & - \\
$\sigma[\mathrm{kHz}]$ & 3.5 & 5.0 & 5.1 & - \\
$s$ & $91.84(19)$ & $92.14(25)$ & $92.16(24)$ & 94.43 \\
$V_{3}[\mathrm{cal} / \mathrm{mole}]$ & $3529(8)$ & $3595(10)$ & $3602(10)$ & $3685(12)$ \\
$F[\mathrm{GHz}]$ & 179.1 & 181.9 & 182.17 & 181.9 \\
$\Varangle(a, i)\left[{ }^{\circ}\right]$ & {$[42.97]$} & {$[42.97]$} & {$[42.97]$} & {$[42.97]$} \\
$\Varangle(b, i)\left[{ }^{\circ}\right]$ & {$[47.03]$} & {$[47.03]$} & {$[47.03]$} & {$[47.03]$} \\
\hline
\end{tabular}


of first order was controlled by a program [10] using diagonalisation of Hamiltonian matrices for lines up to $J=3$. The values are given also in Table 3 . The measured splittings are not sensitive to $\chi_{a b}$.

A comparison of the values of Table 3 shows agreement within the error limits. So the first order analysis is sufficient up to higher $J$ values.

The torsional analysis was based on the mean values of the splittings of the hfs components as given in Table 4. Thus we neglected interaction. We used a program by Woods [11-14] based on the internal axis method.

We tried to fit $w_{1}(s)$, with $s$ the reduced barrier, $I_{x}$ the moment of inertia of the methyl group and $\Varangle(a, i)$ the angle between the $a$ axis and the internal rotation axis. The fit converged, but the correlation is high. In a next step we assumed the angle $\Varangle(a, i)$ from the structure [5], taking the C-C-bond collinear to the internal rotation axis. A rather high value for $I_{\alpha}$ resulted (Tab. 5, column 1). For comparison with literature we finally present in Table 5

[1] G. Bestmann and H. Dreizler, Z. Naturforsch. 37a, 58 (1982).

[2] J. Demaison and H. Dreizler, Z. Naturforsch. 37a, 199 (1982).

[3] J. A. Hardy, A. P. Cox, E. Fliege, and H. Dreizler, Z. Naturforsch. 37a, 1035 (1982).

[4] D. R. Lide, jr., J. Chem. Phys. 30, 37 (1959).

[5] R. H. Schwendeman and G. D. Jacobs, J. Chem. Phys. 36, 245 (1962).

[6] G. K. Pandey and H. Dreizler, Z. Naturforsch. 33a, 204 (1978).

[7] H. D. Rudolph, Z. Angew. Phys. 13,401 (1961).

[8] U. Andresen and H. Dreizler, Z. Angew. Phys. 30, 207 (1970). two fits with $I_{x}$ fixed to $3.1613 \mathrm{amu} \AA^{2}$ [5] and $3.155 \mathrm{amu} \AA^{2}$ and $\Varangle(a, i)=\theta_{\mathrm{m}}=42.97^{\circ}$ [5]. The value $I_{x}=3.155 \mathrm{amu} \AA^{2}$ was determined by a torsion analysis of ethylfluoride [15]. In Table 5 column 4 we repeat the values of [5] for convenience. We favour the values of column 3 , as a comparison with $\mathrm{CH}_{3} \mathrm{CH}_{2} \mathrm{~F}$ is possible. The last column of Table 4 is calculated with the first set of Table 5. The numbers vary slightly with the other sets.

It may be noticed that the $s$ and $V_{3}$ values are lower than in [5]. To our knowledge the barrier is the highest determined from an analysis of splittings of torsion ground state transitions. The errors indicate that we are near the limit of the MWFTtechnique.

\section{Acknowledgements}

We thank the members of our group for help and discussions, the Deutsche Forschungsgemeinschaft and Fonds der Chemie for funds. The calculations were made at the Kiel University computer center.

[9] J. K. Watson, Aspects of Quartic and Sextic Centrifugal Effects on Rotational Energy Levels in Vibrational Spectra and Structure, Vol. 6, J. R. Durig, editor, Elsevier 1977. - V. Typke, Ulm, Programm ZFAP4, private communication.

[10] D. Hübner and M. Stolze, Programm NQHFS, Diplomarbeiten Kiel 1980.

[11] R. C. Woods, J. Mol. Spectr. 21, 4 (1966).

[12] R. C. Woods, J. Mol. Spectr. 22, 49 (1967).

[13] J. Meier, Dissertation 4611, ETH Zürich.

[14] Modified by L. Lutz, Kiel.

[15] E. Fliege, H. Dreizler, J. Demaison, D. Boucher, J. Burie, and A. Dubrulle, J. Chem. Phys. 78, 3541 (1983). 